\title{
Effects of orally administered Euglena gracilis and its reserve polysaccharide, paramylon, on gastric dysplasia in A4gnt knockout mice
}

Masataka lida ${ }^{1,8}$, Mark Joseph Desamero ${ }^{2,3,8}$, Kosuke Yasuda ${ }^{4}$, Ayaka Nakashima $^{4}$, Kengo Suzuki ${ }^{4}$, James Ken Chambers ${ }^{5}$, Kazuyuki Uchida ${ }^{5}$, Ryohei Ogawa ${ }^{6}$, Satoshi Hachimura ${ }^{6}$, Jun Nakayama ${ }^{7}$, Shigeru Kyuwa ${ }^{2}$, Kozue Miura ${ }^{1}$, Shigeru Kakuta ${ }^{2 \varpi} \&$ Kazuhiro Hirayama ${ }^{1 \bowtie}$

Euglena gracilis is widely utilized as food or supplement to promote human and animal health, as it contains rich nutrients. In this study, we administered spray-dried powder of $E$. gracilis and paramylon, $\beta$-glucan stored in E. gracilis cells, to A4gnt knockout (KO) mice. A4gnt KO mice are a mutant mouse model that spontaneously develops gastric cancer through hyperplasia-dysplasiaadenocarcinoma sequence in the antrum of the stomach, and we observed the effects of $E$. gracilis and paramylon on the early involvements of A4gnt $\mathrm{KO}$ mice. Male and female 10-week-old A4gnt $\mathrm{KO}$ mice and their age-matched wildtype C57BL/6J mice were orally administered with $50 \mathrm{mg}$ of $E$. gracilis or paramylon suspended in saline or saline as a control. After 3-week administration, animals were euthanatized and the stomach was examined histopathologically and immunohistochemically. Gene expression patterns of the stomach, which have been reported to be altered with A4gnt KO, and IgA concentration in small intestine were also analyzed with real-time PCR and ELISA, respectively. Administration of Euglena significantly reduced the number of stimulated CD3-positive T-lymphocytes in pyloric mucosa of $A 4 g n t$ KO mice and tend to reduce polymorphonuclear leukocytes infiltration. Euglena administration further downregulated the expression of Il11 and Cxcl1 of A4gnt KO mice. Euglena administration also affected IgA concentration in small intestinal contents of $A 4 g n t$ KO mice. Paramylon administration reduced the number of CD3-positive lymphocytes in pyloric mucosa of A4gnt KO mice, and downregulated the expressions of Il11 and Ccl2 of A4gnt KO mice. Although we found no significant effects on gross and microscopic signs of gastric dysplasia and cell proliferation, the present study suggests that the administration of Euglena and paramylon may ameliorate the early involvements of $A 4 g n t$ mice through the effects on inflammatory reactions in the gastric mucosa. The cancer-preventing effects should be studied with long-term experiments until actual gastric cancer formation.

${ }^{1}$ Laboratory of Veterinary Public Health, Graduate School of Agricultural and Life Sciences, The University of Tokyo, 1-1-1, Yayoi, Bunkyo-ku, Tokyo 113-8657, Japan. ${ }^{2}$ Laboratory of Biomedical Science, Graduate School of Agricultural and Life Sciences, The University of Tokyo, 1-1-1, Yayoi, Bunkyo-ku, Tokyo 113-8657, Japan. ${ }^{3}$ Department of Basic Veterinary Sciences, College of Veterinary Medicine, University of the Philippines Los Baños, 4031 Los Banos, Laguna, Philippines. ${ }^{4}$ euglena Co., Ltd, Minato-ku, Tokyo 108-0014, Japan. ${ }^{5}$ Laboratory of Veterinary Pathology, Graduate School of Agricultural and Life Sciences, The University of Tokyo, 1-1-1, Yayoi, Bunkyo-ku, Tokyo 113-8657, Japan. ${ }^{6}$ Research Center for Food Safety, Graduate School of Agricultural and Life Sciences, The University of Tokyo, 1-1-1, Yayoi, Bunkyo-ku, Tokyo 113-8657, Japan. ${ }^{7}$ Department of Molecular Pathology, Shinshu University School of Medicine, 3-1-1, Asahi, Matsumoto, Nagano 390-8621, Japan. ${ }^{8}$ These authors contributed equally: Masataka lida and Mark Joseph Desamero. ${ }^{\square}$ email: a-skakuta@g.ecc.u-tokyo.ac.jp; akazu@g.ecc.u-tokyo.ac.jp 
Disease prevention and early disease management by food or food components with health-promoting activities along with their nutritional benefits have recently been attracting attention and the term "functional food" is now widely accepted ${ }^{1}$. Various functions have been reported including the reduced risk of coronary heart disease ${ }^{2}$ and the beneficial effect on irritable bowel syndrome ${ }^{3}$. Anti-tumor effect is also one of the growing focused effects. To date, a broad range of examples has been extensively characterized, including eicosapentaenoic acid (EPA), docosahexaenoic acid (DHA), probiotics, phytochemicals, lycopene and $\beta$-glucans ${ }^{4}$.

Euglena gracilis (Euglena) is a unicellular photosynthesizing green alga living in fresh water. In addition to being enriched in nutrients such as vitamins, minerals, amino acids, and fatty acids at high concentrations, it has no cell wall and is highly digestible. Moreover, it has been previously shown to exemplify a number of healthenhancing activities as supported by few studies ${ }^{5-7}$. Therefore, Euglena is now widely utilized as a food or supplement to promote human and animal health. One of the functional ingredients responsible for the health effects of Euglena is paramylon, a $\beta$-1,3-glucan comprising around $20-70 \%$ of Euglena on a dry weight basis. This stored polysaccharide has been reported to possess various effects on the host including immunomodulating effect ${ }^{6,8-13}$.

$\beta$-glucans from multiple sources i.e. mushroom, bacteria, oats, etc. have been well established to exhibit a pronounced anti-inflammatory and anti-tumor efficacies ${ }^{14,15}$, while the anti-tumor activity of paramylon or Euglena is still poorly understood. Previous studies demonstrated that paramylon from Euglena prevents preneoplastic lesions in the mice large intestine, and that another species, Euglena tuba, suppresses metastasis in human lung and breast carcinoma cells. However, it still remains unknown whether paramylon or Euglena have preventive effects on a precursor of gastric cancer ${ }^{16,17}$. Thus, in the present study, we investigated the effects of oral administration of Euglena and paramylon on the early involvements of gastric carcinogenesis in a genetic mouse model.

In this study, we employed A4gnt knockout (KO) mice as an experimental model. A4gnt $\mathrm{KO}$ mice are deficient in $\alpha 1,4-N$-acetylglucosaminyltransferase ( $\alpha 4 \mathrm{GnT}$ ), a glycosyltransferase responsible for the biosynthesis of $\alpha 1,4-N$-acetylglucosamine capped $O$-glycans ( $a$ GlcNAc) in gastric gland mucin ${ }^{18}$. This mutant mouse model spontaneously develops gastric cancer through hyperplasia-dysplasia-adenocarcinoma sequence in the antrum of the stomach ${ }^{19}$. At 10-20 weeks of age, the age used in the present study, A4gnt KO mice show precancerous lesion of gastric cancer, dysplasia.

In addition, we also measured the concentration of $\operatorname{Ig} \mathrm{A}$ in tissue and contents of small intestine of the mice, because many $\beta$-glucans have been reported to have immunostimulatory effects through host innate immunity ${ }^{8}$.

\section{Results}

Effects of Euglena and paramylon on the gastric mucosal thickness. The body weight change and gross conditions of the mice in all groups were comparable. A4gnt $\mathrm{KO}$ mice showed significantly thick gastric mucosa in the antrum of the stomach compared with wildtype mice (Fig. 1, Table 1). Administration of Euglena and paramylon did not visibly affect the thickness of the pyloric mucosa. The thickness of gastric mucosa of KO control, KO euglena and $\mathrm{KO}$ paramylon groups were $418.6 \pm 21.4,421.5 \pm 17.5$ and $421.8 \pm 22.7 \mu \mathrm{m}$, respectively (Fig. 1E).

Effects of Euglena and paramylon on the infiltration of inflammatory cells. In A4gnt KO mice, it has been strongly suggested that infiltration of inflammatory cells to gastric mucosa initiate the precursor lesion and progress the cascade to differentiated-type gastric cancer ${ }^{19}$. Therefore, we histopathologically assessed the number of infiltrating polymorphonuclear leukocytes (PMNLs) and CD3-positive lymphocytes. The number of PMNLs (Fig. 2A-D, arrowheads) in the pyloric mucosa of A4gnt KO mice was significantly higher than that in wildtype mice (Fig. 2E, Table 1), and the number was slightly reduced by Euglena administration $(P=0.092)$, thus making the difference between $\mathrm{KO}$ euglena group and wildtype euglena group not significant (Table 1). On the other hand, the effect of paramylon on the infiltration of PMNLs was not obvious. The number of infiltrating CD3-positive T-lymphocytes was significantly higher in $\mathrm{KO}$ mice compared with in wildtype mice, and significantly reduced by the administration of Euglena and paramylon (Fig. 3, Table 1, Supplemental Fig. 1A-C).

Effects of Euglena and paramylon on the proliferation of S-phase cells and angiogenesis. To investigate the possible effects of Euglena and paramylon administration on the cell proliferation and angiogenesis, important mechanisms in the gastric carcinogenesis of A4gnt $\mathrm{KO}$ mice, we then performed BrdU-pulse labeling and CD31 immunohistochemistry (Figs. 4 and 5). The numbers of BrdU- and CD31-positive cells were significantly higher in A4gnt $\mathrm{KO}$ mice (Figs. 4E and 5E, Table 1). However, the effects of Euglena and paramylon administration were comparable with the corresponding control counterparts (Figs. 4 and 5).

Effects of Euglena and paramylon on the gene expression profiles of several cytokines. Next, we performed quantitative RT-PCR analysis of genes encoding inflammatory chemokine ligands, proinflammatory cytokines, and growth factors, which are either upregulated or downregulated in the A4gnt KO at 10 weeks of age ${ }^{19}$. Quantitative RT-PCR analysis revealed marked changes of the mean expression levels of genes examined except for IllO in A4gnt $\mathrm{KO}$ mice compared with those in wildtype mice (Fig. 6, Table 1). In consonance with previous finding ${ }^{19}$, the expressions of Illb, Il11, Cxcl1, Ccl2, Hgf, Fgf7 and Ptgs2 were significantly higher in A4gnt $\mathrm{KO}$ mice than in wildtype mice, and Egf was significantly lower in A4gnt KO mice. Administration of Euglena significantly downregulated the expressions of Il11 and Cxcl1 in A4gnt KO mice when compared with saline-treated A4gnt KO mice whereas the gene transcription level of Cxcll between A4gnt $\mathrm{KO}$ and wildtype mice was insignificant. On the other hand, the gene expression of $\mathrm{Ccl} 2$ of $\mathrm{KO}$ euglena group was also appreciably lower than that of $\mathrm{KO}$ control group, though the difference did not attain statistical significance. Meanwhile, administration of paramylon to A4gnt $\mathrm{KO}$ mice also significantly reduced $I l 11$ and $\mathrm{Ccl} 2$ expressions. In the 


\section{Wildtype control}

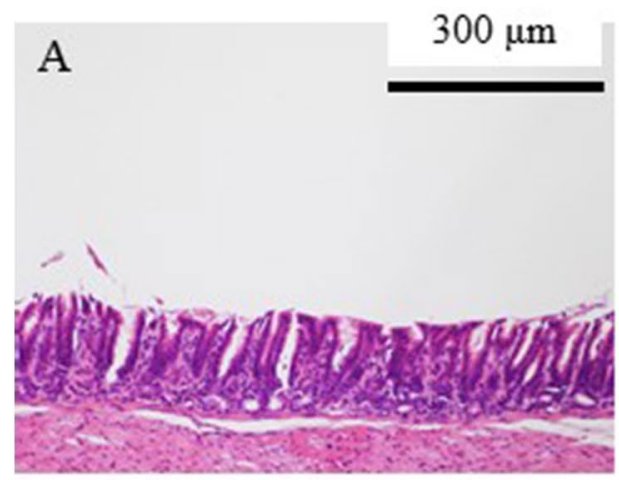

A4gnt $\mathrm{KO}$ euglena

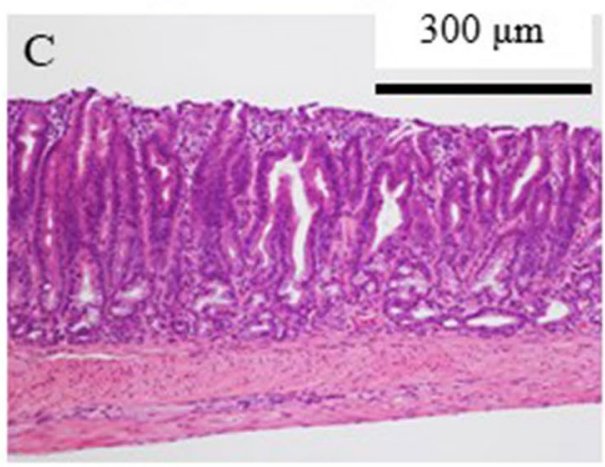

A4gnt KO control

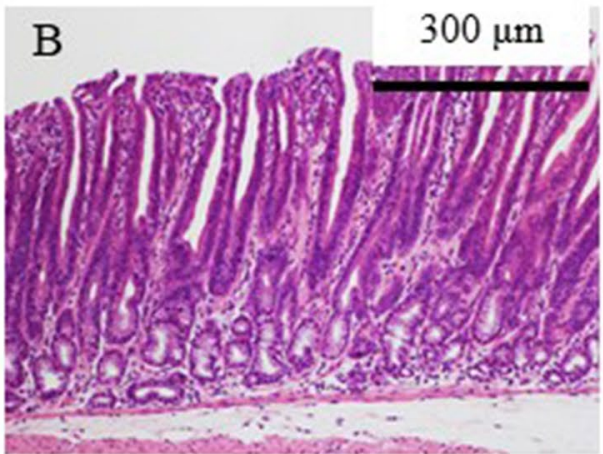

A4gnt KO paramylon

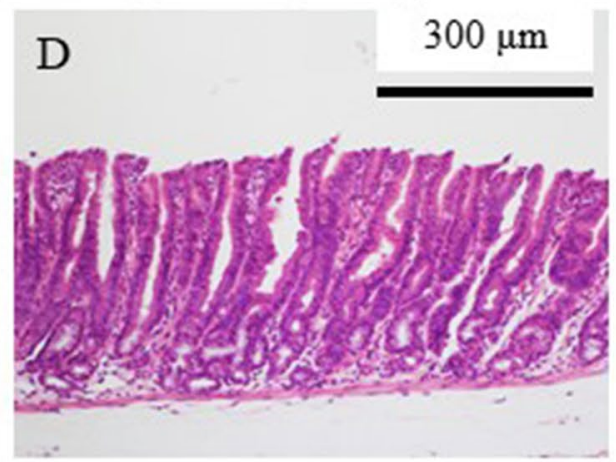

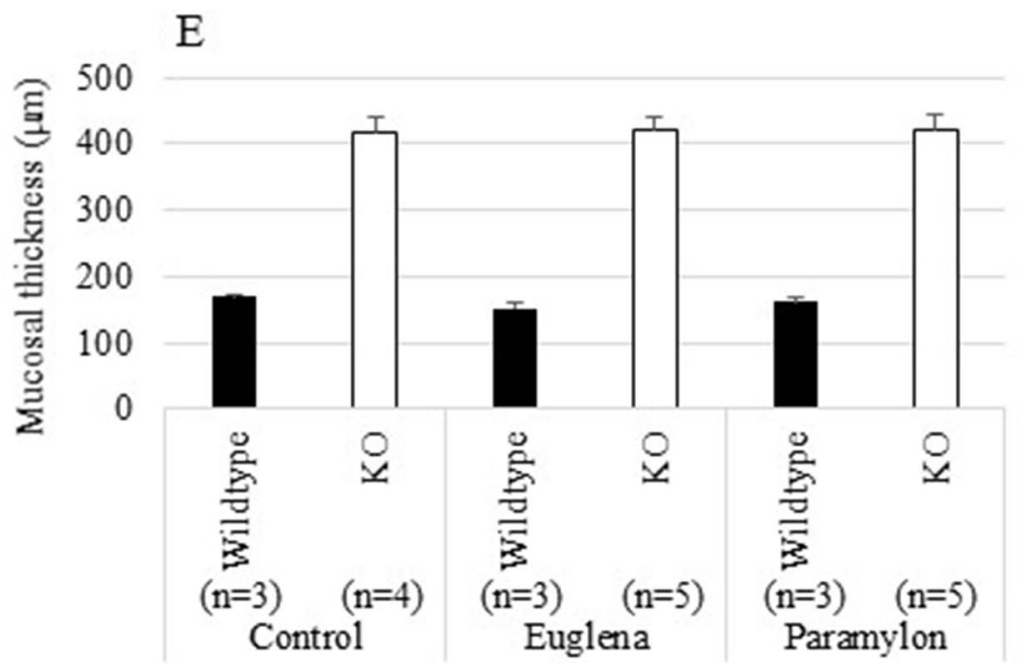

Figure 1. Histopathology of pyloric mucosa of A4gnt $\mathrm{KO}$ and wildtype mice. (A) Wildtype mouse administered saline. (B) A4gnt KO mouse administered saline. (C) A4gnt $\mathrm{KO}$ mouse administered $50 \mathrm{mg} /$ day of Euglena. (D) A4gnt KO mouse administered $50 \mathrm{mg} /$ day of paramylon. (E) Mean $\pm \mathrm{SD}$ of thickness of pyloric mucosa. The mean thickness of gastric mucosa of 13-week-old A4gnt KO mice was significantly thicker compared with that of wildtype mice. Administration of Euglena and paramylon did not affect the thickness of the pyloric mucosa.

wildtype control mice, neither Euglena nor paramylon solicited any substantial difference in the resulting gene transcription profile of all examined inflammation-associated genes.

Effects of Euglena and paramylon on IgA production in small intestine. The concentration of IgA in the small intestinal contents of A4gnt $\mathrm{KO}$ mice tended to increase following Euglena administration (Fig. 7, 


\begin{tabular}{|c|c|c|c|c|c|c|c|c|c|c|c|c|c|c|}
\hline Groups & Thickness & PMNL & CD3 & BrdU & CD31 & Il11 & Illo & $H g f$ & $I l 1 b$ & Cxcl1 & $\mathrm{Ccl} 2$ & Egf & $F g f 7$ & Ptg2 \\
\hline \multicolumn{15}{|c|}{ A4gnt KO vs. wild type } \\
\hline Control & $* * *$ & * & $* * *$ & $* *$ & $* * *$ & $* * *$ & ns & $* * *$ & $* * *$ & $* * *$ & $* * *$ & $* * *$ & $* *$ & * \\
\hline Euglena & $* * *$ & ns & $* * *$ & * & * & ns & ns & $* * *$ & ns & ns & * & $* * *$ & $* *$ & * \\
\hline Paramylon & $* * *$ & $*$ & * & $* *$ & * & * & ns & $* * *$ & * & * & * & $* * *$ & * & $* *$ \\
\hline \multicolumn{15}{|c|}{ Control vs. euglena } \\
\hline A4gnt KO & ns & ns & $* *$ & ns & ns & ** & ns & ns & ns & $*$ & ns & ns & ns & ns \\
\hline Wild type & ns & ns & ns & ns & ns & ns & ns & ns & ns & ns & ns & ns & ns & ns \\
\hline \multicolumn{15}{|c|}{ Control vs. paramylon } \\
\hline A4gnt KO & ns & $\mathrm{ns}$ & ** & ns & $\mathrm{ns}$ & $*$ & ns & $\mathrm{ns}$ & $\mathrm{ns}$ & ns & * & ns & $\mathrm{ns}$ & $\mathrm{ns}$ \\
\hline Wild type & ns & ns & ns & ns & ns & ns & ns & ns & ns & ns & ns & ns & ns & ns \\
\hline \multicolumn{15}{|c|}{ Euglena vs. paramylon } \\
\hline A4gnt KO & ns & ns & ns & ns & ns & ns & ns & ns & ns & ns & ns & ns & ns & $\mathrm{ns}$ \\
\hline Wild type & ns & ns & ns & ns & ns & ns & ns & ns & ns & ns & ns & ns & ns & ns \\
\hline
\end{tabular}

Table 1. Summary of statistical analysis. Tukey's honest significance test using R software ver. 3.6.2 ${ }^{28}$ was used to analyze the differences and measure the significance. ${ }^{\star * *} P$ value $<0.001,{ }^{* *} P$ value $<0.01,{ }^{\star} P$ value $<0.05$, ns $P$ value $>0.05$.

Table 1). Nevertheless, we could not find any difference in the concentration of IgA in the small intestinal tissue among the examined treatment groups.

\section{Discussion}

In the present study, we investigated the protective effects of oral administration of Euglena and paramylon on gastric dysplasia development using A4gnt KO mice, a unique animal model for gastric cancer that spontaneously develops differentiated-type gastric adenocarcinoma in a hyperplasia-dysplasia-carcinoma sequence ${ }^{19}$.

The histopathological and immunohistochemical investigation demonstrated that administration of Euglena significantly reduced the elevated infiltration of CD3-positive T-lymphocytes in the pyloric mucosa of A4gnt KO mice. Although not statistically significant with the corresponding KO control group, Euglena supplementation slightly inhibited the A4gnt KO-induced sequestration of PMNLs. In support of this data, we then demonstrated that Euglena administration significantly suppressed $I l 11$ and Cxcl1 gene expressions, together with a tendency to reduce $C c l 2$ expression. IL-11, a member of the IL- 6 family, promotes the progression of chronic inflammation to gastric carcinoma via gp130-mediated activation of STAT3 and STAT1 signaling in human gastric carcinogenesis ${ }^{20}$. On the other hand, Cxcl1-expressing cancer cells stably exhibited an increased migration and invasion abilities ${ }^{21}$. Owing to the notion that chronic inflammation is the first crucial event in carcinogenesis ${ }^{22}$ that is intricately linked with the continuum of precancerous cascade (metaplasia, dysplasia) to invasive carcinoma ${ }^{21,23}$, our findings altogether suggest that Euglena may attenuate the early involvement of gastric carcinogenesis in A4gnt $\mathrm{KO}$ mice through suppression of mucosal inflammation. Moderate stimulation of IgA production in small intestine also partly supports the influence of Euglena on the host immunological condition.

Paramylon, one of the major components of Euglena, also significantly suppressed the infiltration of CD3positive T-lymphocytes and downregulated the expression of Il11 gene in pyloric mucosa of A4gnt KO mice. Although the reduction of the gene expression of $\mathrm{Cxcl} 1$ by paramylon, unlike Euglena, was not statistically significant, paramylon significantly reduced $\mathrm{Ccl} 2$ expression. These results indicate the anti-inflammatory effects of paramylon.

Paramylon is a member of $\beta$-glucans and various effects of $\beta$-glucans on the host have been reported ${ }^{14,15}$. For example, marked suppressive effects of laminaran, a $\beta$-glucan derived from brown algae, Eisenia bicyclis, on the development of gastric dysplasia is reported using the same A4gnt KO mouse model ${ }^{24}$. However, the effects of Euglena and paramylon were different from those of laminaran. For example, laminaran suppressed the expression of Il10, while Euglena and paramylon did not. Paramylon is $\beta$-1,3-glucan, consists of only glucose (mean, 700 glucose molecules) and a high molecular weight $(\sim 500 \mathrm{kDa})$ insoluble $\beta$-glucan ${ }^{8}$, while laminaran is low molecular weight $(\sim 5 \mathrm{kDa})$ and soluble. The differences in their structure, such as size of molecule, solubility, types and frequency of side chains, might affect the types and strength of physiological functions. Although it is reported that the effects of laminaran is mediated by dectin-1, the present study did not give enough information to elucidate the mechanism of action of Euglena and paramylon. Future studies to elucidate the mode of action, e.g. experiments with inhibitor of receptors, are needed.

In the present study, we analyzed the parameters which have been reported to be affected by A4gnt deficiency ${ }^{19}$, because the aim of this study is to investigate the effects of Euglena and paramylon on the early involvements of the A4gnt KO gastric cancer model. However, it is also possible that Euglena and paramylon showed, indirectly, anti-inflammatory effects through the other factors. Further experiments studying the expression of other cytokines would be interesting.

Contrary to expectation that the major mechanism of the effects of Euglena is due to paramylon, the effects of Euglena were at the same level, or even stronger, when compared with the effects of paramylon. As we administered the same dose of Euglena and paramylon to A4gnt KO mice, and paramylon consists 30-40\% of dry 

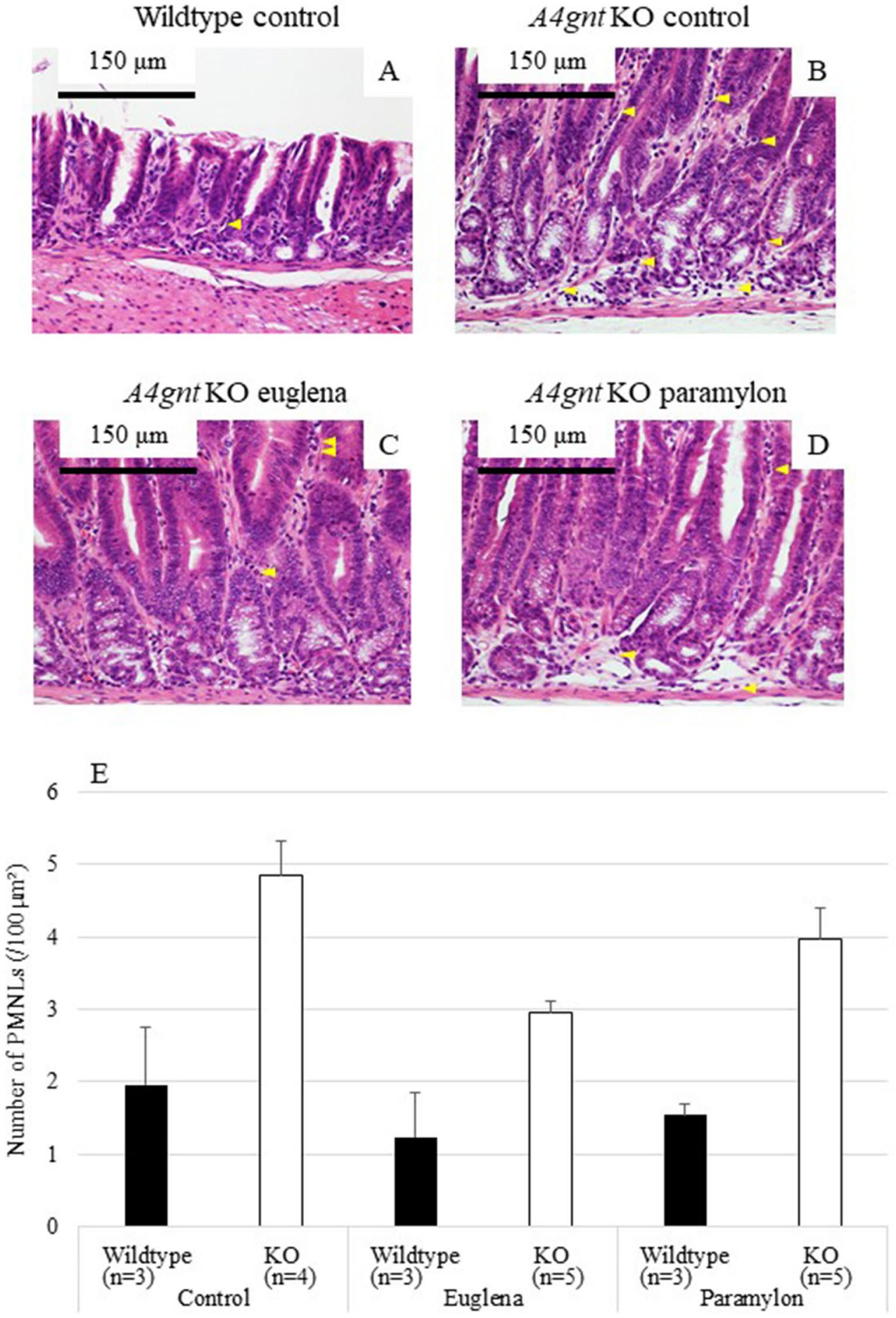

Figure 2. Numbers of PMNLs in pyloric mucosa of A4gnt $\mathrm{KO}$ and wildtype mice. (A) Wildtype mouse administered saline. (B) A4gnt KO mouse administered saline. (C) A4gnt KO mouse administered $50 \mathrm{mg} /$ day of Euglena. (D) A4gnt KO mouse administered $50 \mathrm{mg} /$ day of paramylon. Arrowheads indicate PMNLs. (E) Mean \pm SD of numbers of PMNLs in pyloric mucosa. The mean number of PMNLs in gastric mucosa of $A 4 g n t$ $\mathrm{KO}$ mice was significantly more than that of wildtype mice. Administration of Euglena significantly reduced the number of PMNLs. be important for the anti-carcinogenic effects, which can be harnessed for commercial use ${ }^{25}$. 


\section{Wildtype control}

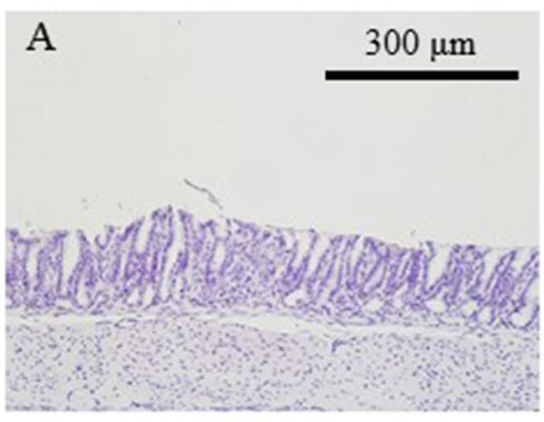

A4gnt $\mathrm{KO}$ euglena

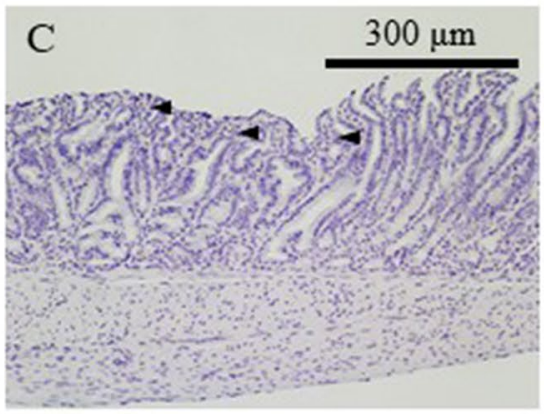

A4gnt KO control

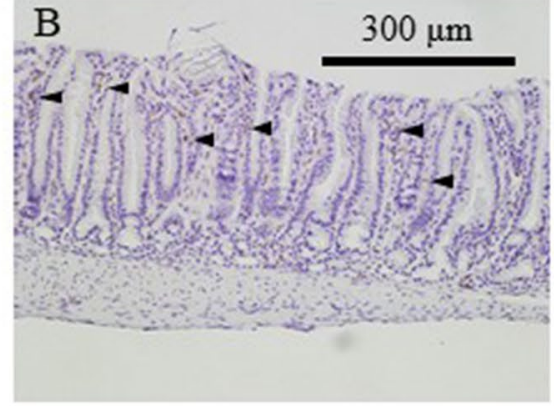

A4gnt KO paramylon

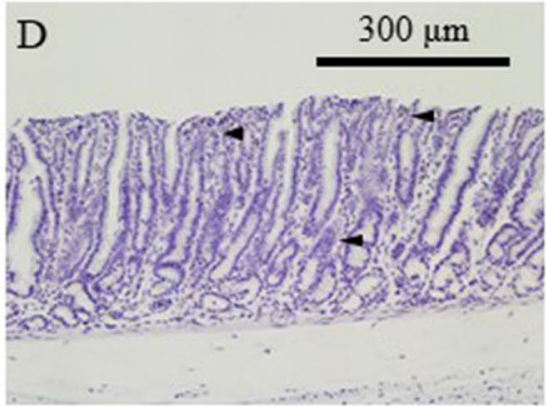

$\mathrm{E}$

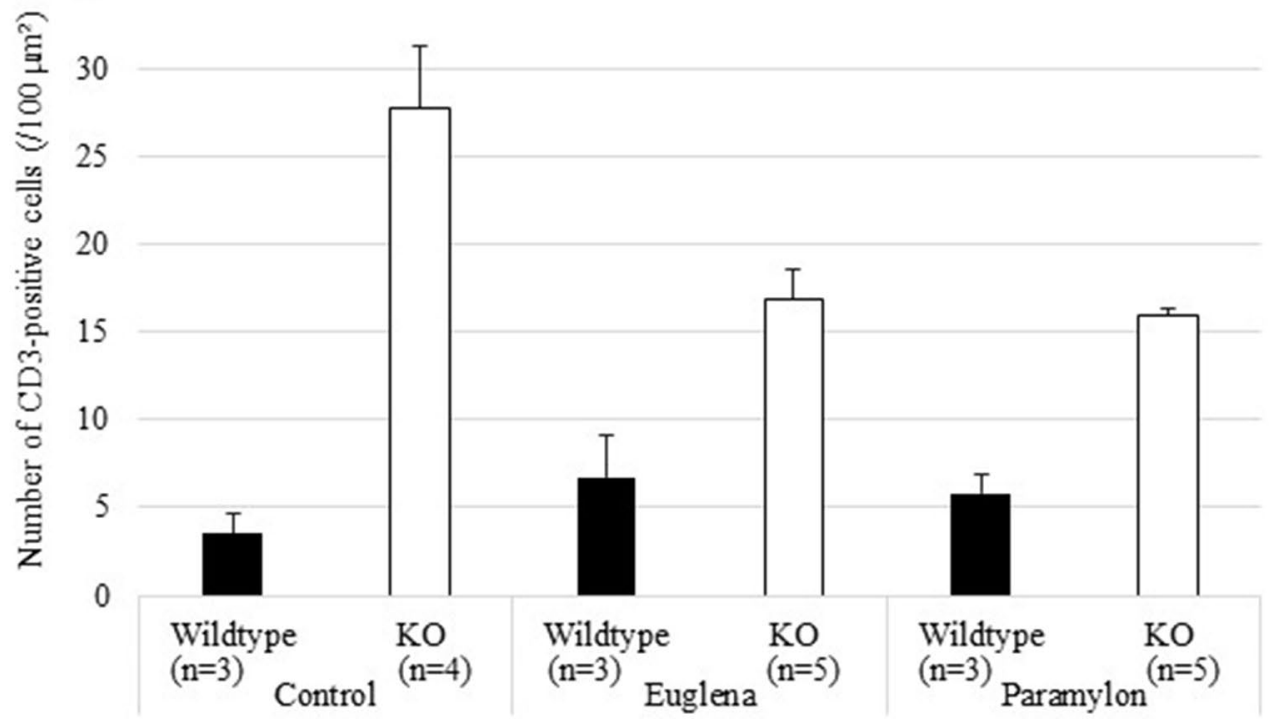

Figure 3. Numbers of CD3-positive lymphocytes in gastric mucosa of A4gnt $\mathrm{KO}$ and wildtype mice. (A) Wildtype mouse administered saline. (B) A4gnt KO mouse administered saline. (C) A4gnt KO mouse administered $50 \mathrm{mg} /$ day of Euglena. (D) A4gnt $\mathrm{KO}$ mouse administered $50 \mathrm{mg} /$ day of paramylon. CD3-positive cells were stained brown by diaminobenzidine tetrahydrochloride. (E) Mean \pm SD of numbers of CD3-positive cells in pyloric mucosa. Arrowheads indicate CD3-positive cells. The mean number of CD3-positive cells in gastric mucosa of A4gnt KO mice was significantly more than that of wildtype mice. Administration of Euglena and paramylon significantly reduced the number of CD3-positive cells of A4gnt $\mathrm{KO}$ mice compared with those in control group administered with saline. 
Wildtype control

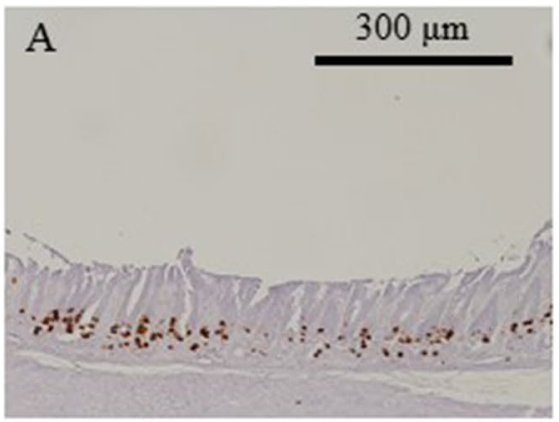

A4gnt $\mathrm{KO}$ euglena

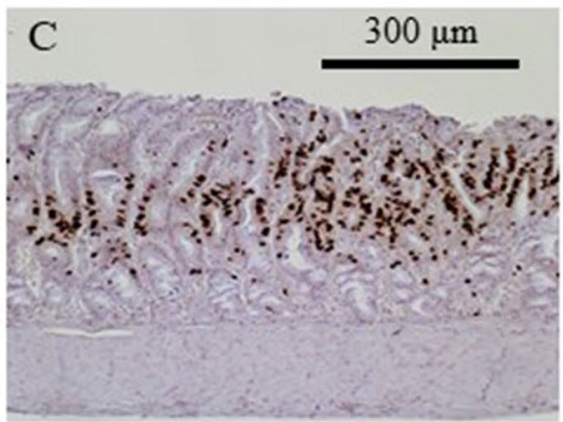

A4gnt KO control

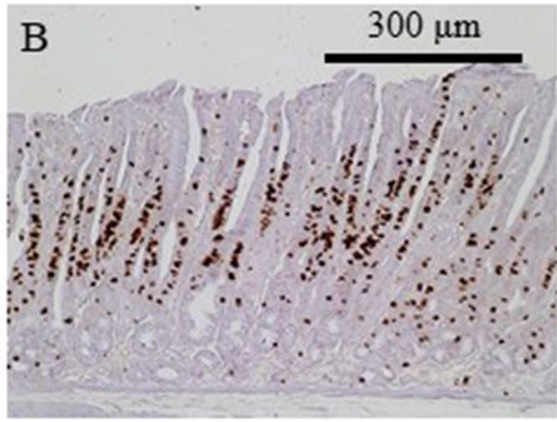

A4gnt KO paramylon

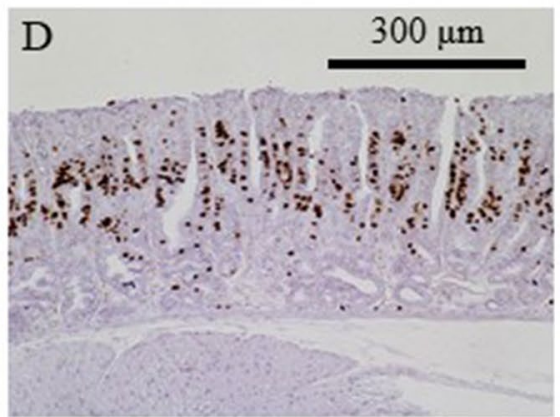

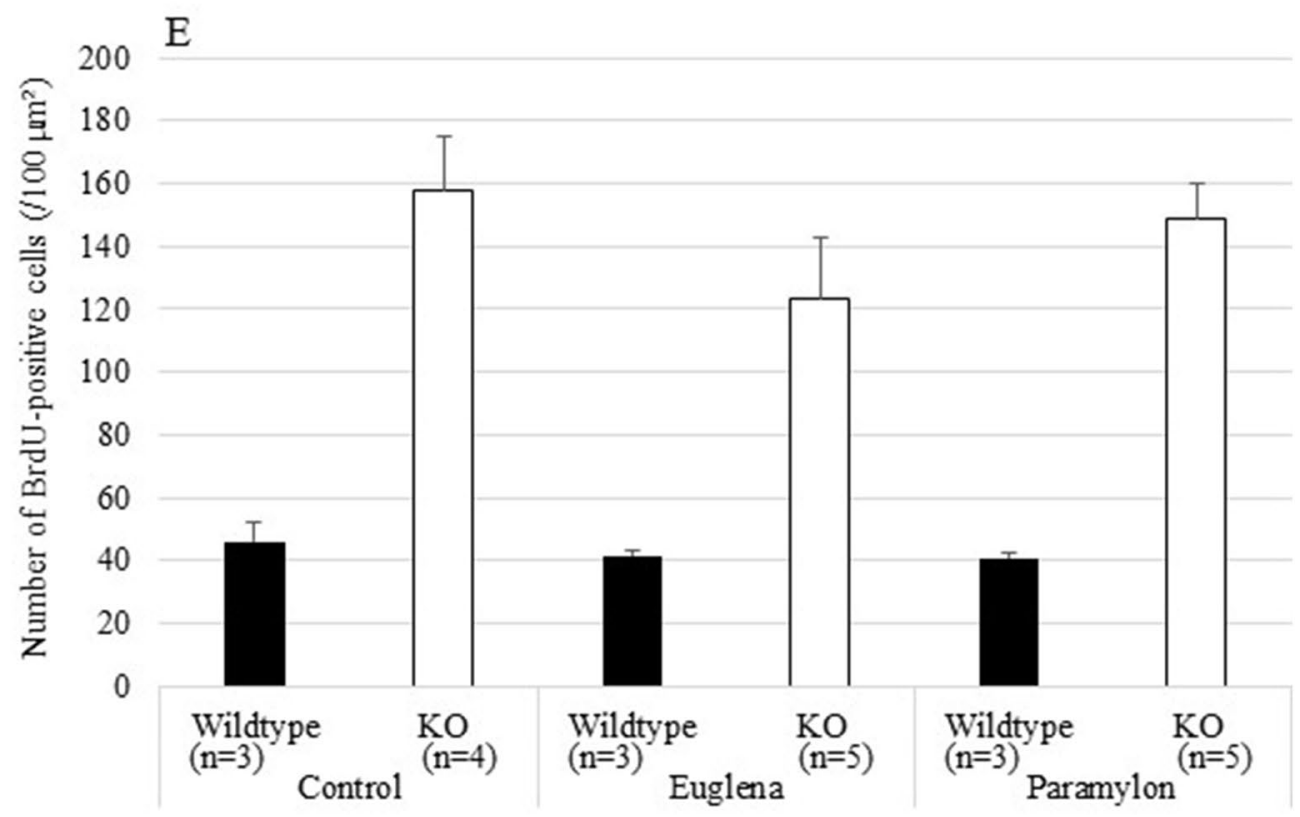

Figure 4. Numbers of BrdU-positive cells in gastric mucosa of A4gnt $\mathrm{KO}$ and wildtype mice. (A) Wildtype mouse administered saline. (B) A4gnt KO mouse administered saline. (C) A4gnt KO mouse administered $50 \mathrm{mg} /$ day of Euglena. (D) A4gnt $\mathrm{KO}$ mouse administered $50 \mathrm{mg}$ /day of paramylon. BrdU-positive cells were stained brown by diaminobenzidine tetrahydrochloride. (E) Mean \pm SD of numbers of BrdU-positive cells in pyloric mucosa. The mean numbers of BrdU-positive cells in gastric mucosa of A4gnt KO mice was significantly more than that of wildtype mice. Administration of Euglena and paramylon did not affect the numbers of these cells. 
Wildtype control

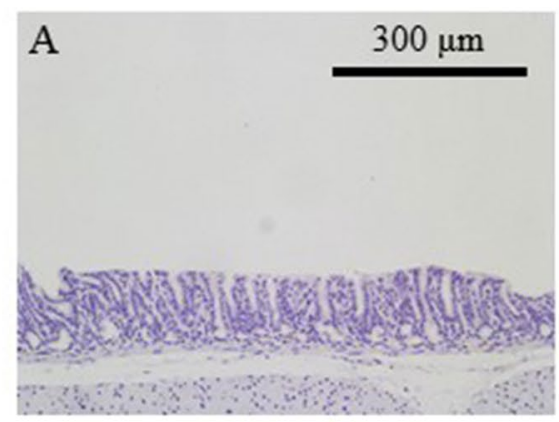

A4gnt KO euglena

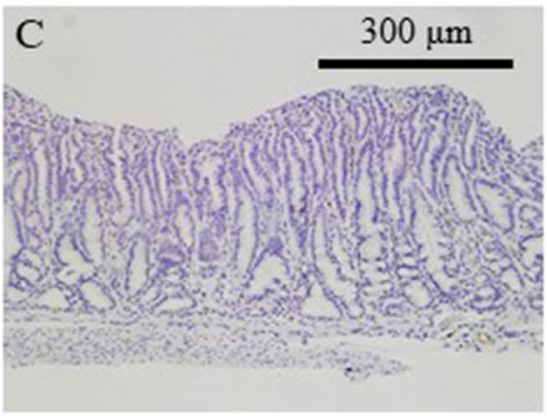

A4gnt KO control

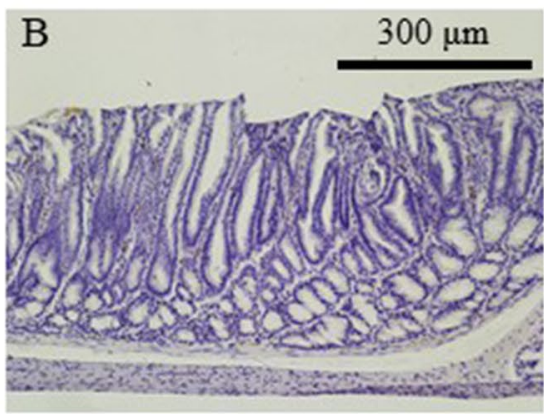

A4gnt KO paramylon

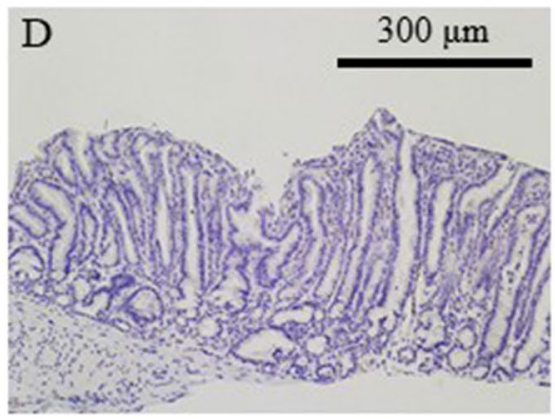

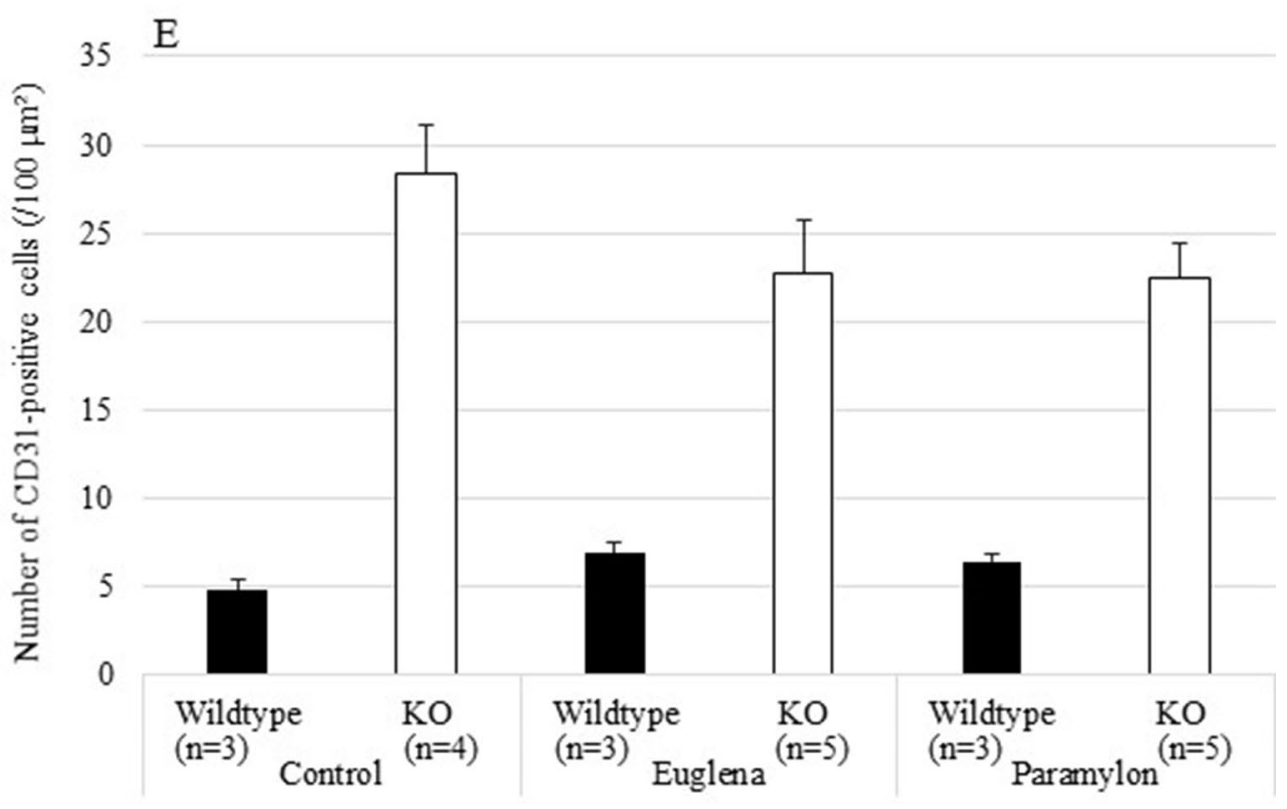

Figure 5. Numbers of CD31-positive cells in gastric mucosa of A4gnt $\mathrm{KO}$ and wildtype mice. (A) Wildtype mouse administered saline. (B) A4gnt KO mouse administered saline. (C) A4gnt $\mathrm{KO}$ mouse administered $50 \mathrm{mg} /$ day of Euglena. (D) A4gnt $\mathrm{KO}$ mouse administered $50 \mathrm{mg} /$ day of paramylon. CD31-positive cells were stained brown by diaminobenzidine tetrahydrochloride. (E) Mean \pm SD of numbers of CD31-positive cells in pyloric mucosa. The mean numbers of CD31-positive cells in gastric mucosa of A4gnt KO mice was significantly more than that of wildtype mice. Administration of Euglena and paramylon did not affect the numbers of these cells. 

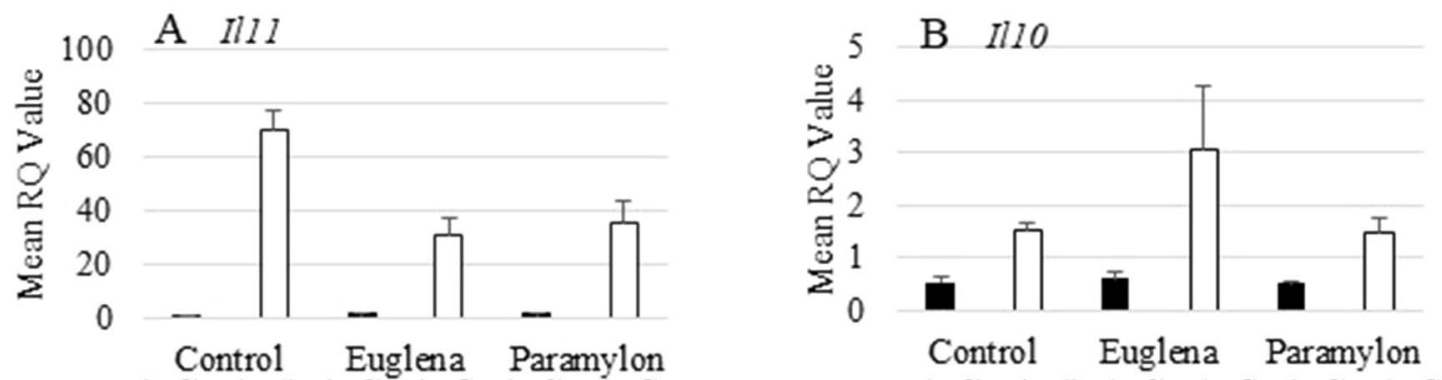

$(n=3) \quad(n=4) \quad(n=3) \quad(n=5) \quad(n=3) \quad(n=5)$

$(n=3) \quad(n=4) \quad(n=3) \quad(n=5) \quad(n=3) \quad(n=5)$
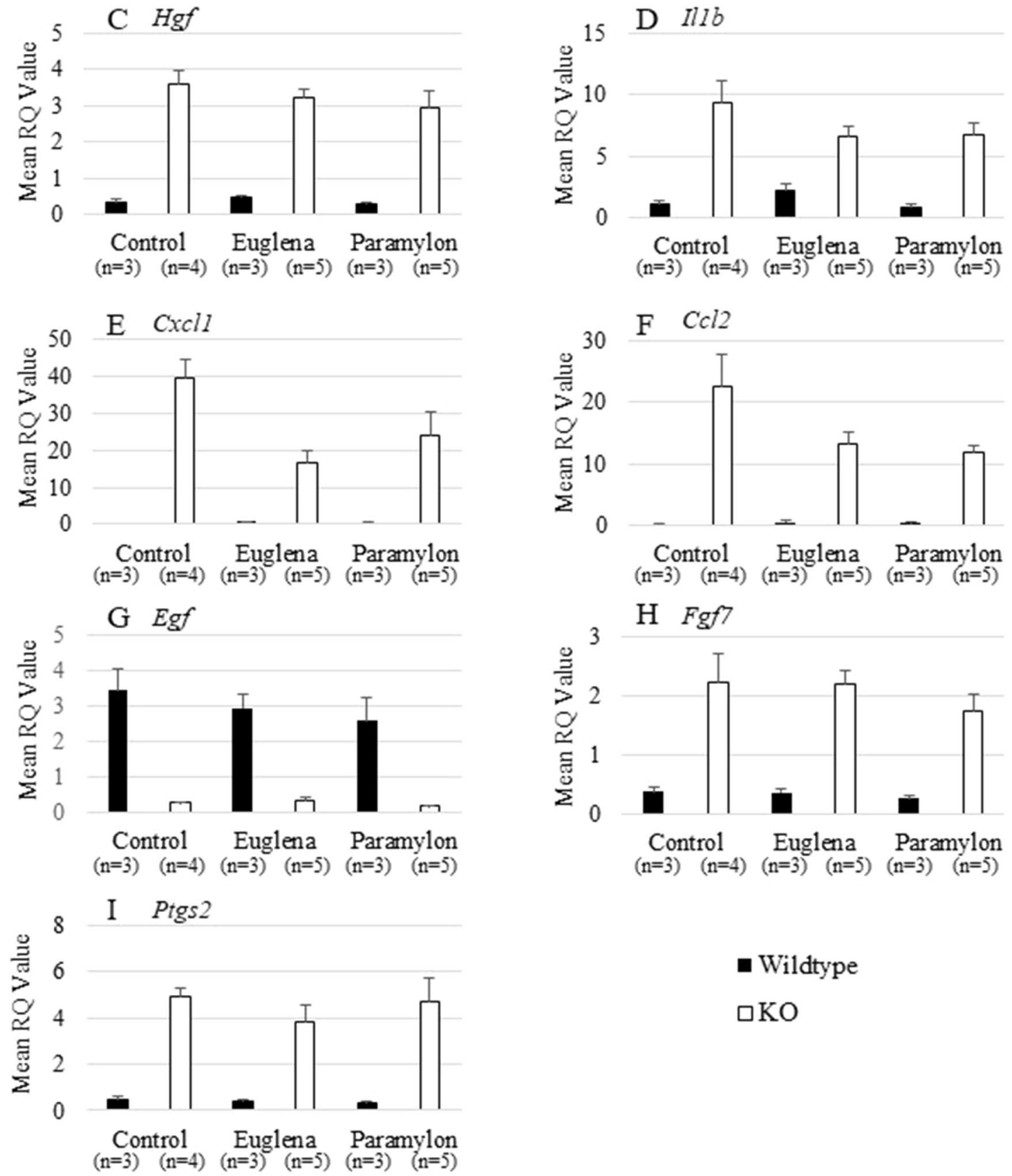

\section{- Wildtype $\square \mathrm{KO}$}

Figure 6. Mean gene expression levels of selected cytokines whose expressions are reported to be substantially altered following A4gnt KO-induced gastric carcinogenesis. (A) Il1, (B) Il10, (C) Hgf, (D) Il11b, (E) Cxcl1, (F) Ccl2, (G) Egf, (H) Fgf7, (I) Ptgs2. The expressions of gene studied were significantly higher in A4gnt KO mice than those in wildtype mice except for the expression of Egf whose expression was significantly lower in A4gnt KO mice. Administration of Euglena and paramylon significantly reduced the expressions of Il11, Cxcl1 and Ccll2. 
A

Contents

16

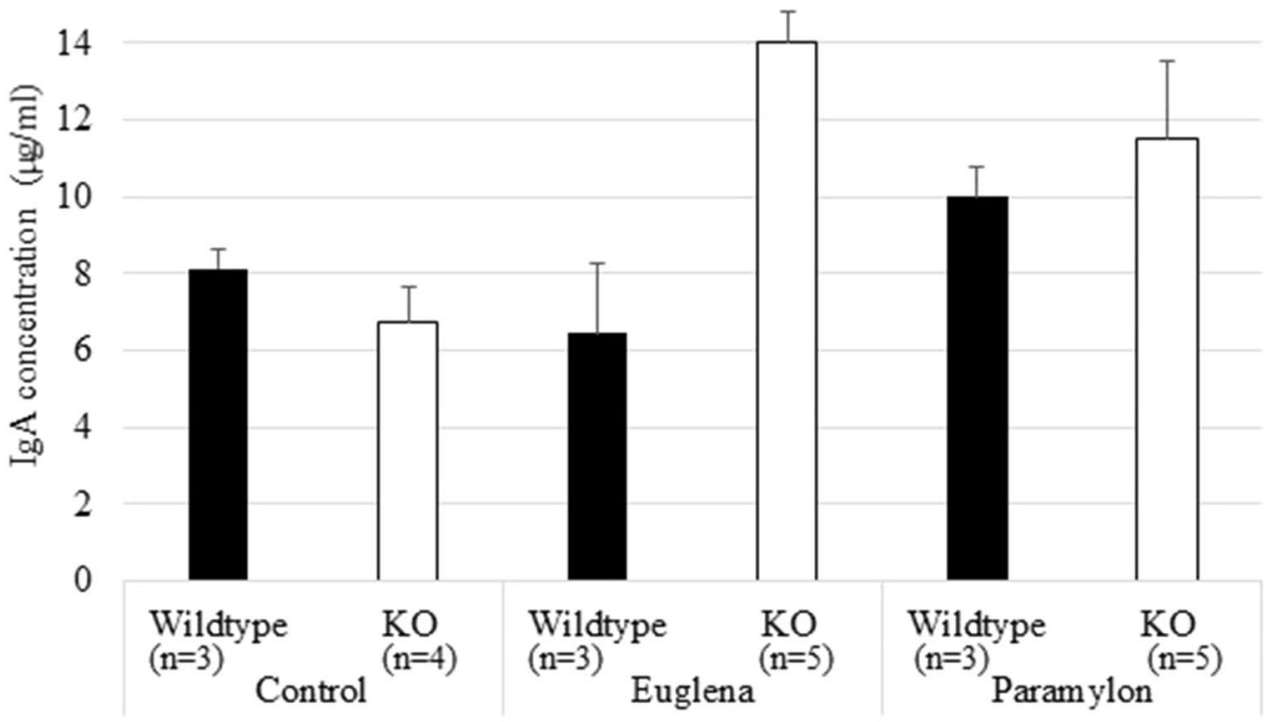

B

Tissue

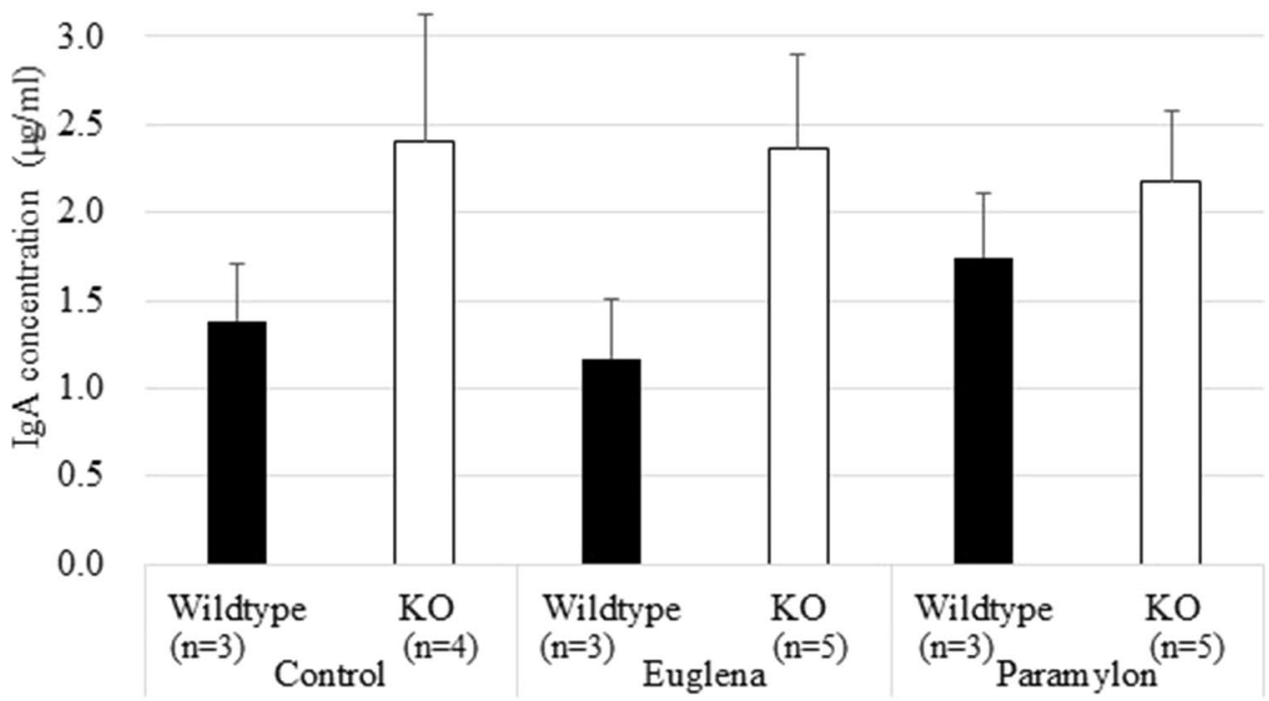

Figure 7. Concentrations of IgA in small intestinal contents and tissue. (A) Contents. (B) Tissue. In the small intestinal contents, IgA concentration significantly increased after 3 weeks administration of Euglena and slightly increased by paramylon administration. In the small intestinal tissue, IgA concentration tend to increase in A4gnt $\mathrm{KO}$ mice.

Cancer survival rate is still closely related to the stage of cancer detection. Therefore, it is important, along with early detection, to control the onset and progression of the cancer by taking functional foods routinely. In the present study, we demonstrated the anti-inflammatory effects of Euglena and its component, paramylon in A4gnt KO mice. As the chronic inflammation is one of the early involvements of this spontaneous gastric cancer model, it is plausible that Euglena and paramylon may restrain the progression of early stage of hyperplasia-dysplasiaadenocarcinoma sequence of the A4gnt $\mathrm{KO}$ mice through ameliorating the ensuing inflammation in the gastric mucosa. However, as A4gnt KO mice develop only precancerous lesion, gastric dysplasia, at the age we used in the present study, and we did not investigate the effects of Euglena and paramylon on gastric cancer in this study, it is impossible to discuss about cancer-preventing effects of these compounds. Further experiments with longer 
period for at least 50 weeks until the formation of gastric cancer are needed to prove the anti-carcinogenic effects of these compounds. We are conducting the long-term experiment with this model to investigate the effects of these compounds on cancer formation. In addition, the administration of Euglena and paramylon did not appear to have remarkable effects on the thickness of pyloric mucosa and the number of BrdU- and CD31-positive cells. Three-week administration may not be long enough to reduce cell proliferation and angiogenesis. Administration from earlier stage might be warranted to prove the effects of these compounds. Application of other cancer models showing gastric precancerous lesions ${ }^{26}$ should also be discussed.

\section{Methods}

Animals. Male and female 10-week-old C57BL/6J-background A4gnt KO mice ${ }^{19}$, which exhibited a lowgrade gastric dysplasia, were bred in the Laboratory of Biomedical Science, Graduate School of Agricultural and Life Sciences, The University of Tokyo. Their age-matched syngenic wildtype C57BL/6J mice were purchased as control animals from CLEA Japan, Inc., (Japan). Animals were housed in standard polycarbonate cages and maintained under $12 \mathrm{~h}$ light/dark cycle (8:00 a.m.: 8:00 p.m.). Rodent chow (CMF; Oriental Yeast Co., Ltd, Japan) and water were given ad libitum. All experimental procedures were performed in accordance with the guidelines and approval of the Institutional Animal Care and Use Committee, Graduate School of Agriculture and Life Sciences, The University of Tokyo (Approval No. P18-053). The experiments were performed also in compliance with the ARRIVE guidelines 2.0.

Preparation of Euglena and paramylon. Spray-dried powder of Euglena and paramylon were obtained from euglena Co., Ltd. (Japan). The nutritional analysis results of Euglena powder were carbohydrates $45.5 \%$, protein $32.3 \%$, and lipid $14.7 \%$. Approximately $70-80 \%$ of the carbohydrate content was paramylon. Paramylon is a $3-1,3-\mathrm{D}$-glucan isolated from Euglena, and its content varies with culture method ${ }^{27}$. Paramylon powder was prepared as previously described ${ }^{6}$. In brief, cultured $E$. gracilis cells were collected by continuous centrifugation and washed with water. After suspending in water, the cells were broken down by sonication and the insoluble fraction containing paramylon was collected. To remove the lipid and protein, the crude paramylon preparation was treated with $1 \%$ sodium dodecyl sulfate (SDS) solution at $95{ }^{\circ} \mathrm{C}$ for $1 \mathrm{~h}$, and then at $50{ }^{\circ} \mathrm{C}$ for $30 \mathrm{~min}$ with $0.1 \%$ SDS. Paramylon was collected by centrifugation, and further refined by repeated washing with water, acetone, and ether, sequentially. The obtained paramylon consists of $95.9 \%$ carbohydrates, $3.3 \%$ moisture, $0.5 \%$ lipids, and $0.4 \%$ ash, and the survival rates of protein are less than $0.1 \%$.

Treatment groups. A4gnt $\mathrm{KO}$ and wildtype animals were randomly assigned into 3 groups, i.e. control group, euglena group and paramylon group. Control groups were administered $0.4 \mathrm{ml}$ of saline. Euglena groups were administered $50 \mathrm{mg}$ of euglena suspended in $0.4 \mathrm{ml}$ of saline. Paramylon groups were administered $50 \mathrm{mg}$ of paramylon suspended in $0.4 \mathrm{ml}$ of saline. All treatments were given daily via oral gavage for 21 consecutive days. The number of animals were 3, 4, 3, 5, 3 and 5 in wildtype control, $\mathrm{KO}$ control, wildtype euglena, $\mathrm{KO}$ euglena, wildtype paramylon and $\mathrm{KO}$ paramylon groups, respectively.

Histopathology of pyloric mucosa. One hour prior to sacrifice, animals were injected intraperitoneally with bromodeoxyuridine (5-bromo-2-deoxyuridine, BrdU) solution (10 mg/kg) to label proliferating cells in the $\mathrm{S}$-phase of the cell cycle ${ }^{19}$. Animals were euthanatized by cervical dislocation and stomach tissues along with a small portion of the duodenum were harvested. Stomach was cut longitudinally along the greater curvature, washed with $1 \times$ PBS and divided into half for subsequent histopathological, immunohistochemical and gene expression analyses. A half of the stomach was fixed in $10 \%$ buffered formaldehyde for at least $48 \mathrm{~h}$ and subjected to routine paraffin technique. Four-micrometer sections were then prepared and stained with standard H\&E. Pyloric mucosal thickness was measured from the base of the gastric mucosal layer up to the highest tip of a properly oriented epithelium at three different areas of the pyloric region. Mean of triplicate measurements for each animal was obtained. For determining the number of infiltrating granulocytes, a defined $100 \mu \mathrm{m}^{2}$ area with the highest cell density along the pyloric region was counted and a mean of triplicate measurements was then obtained.

CD3, CD31 and BrdU immunohistochemistry. Formalin-fixed and paraffin-embedded (FFPE) tissue sections $(4 \mu \mathrm{m})$ were deparaffinized by xylene for 4 times, rehydrated with increasing grades of alcohol $(100,95$, 90, 80 and 70\%), and washed with Tris-buffered saline (TBS; $0.1 \mathrm{M}, \mathrm{pH}$ 7.4) for 3 times. Prior to incubation with primary antibodies, antigen retrieval was carried out using $4 \mathrm{~N} \mathrm{HCl}$ followed by digestion with $0.5 \%$ trypsin (Thermo Fisher Scientific Inc., USA) for $30 \mathrm{~min}$ at $37^{\circ} \mathrm{C}$ for anti-BrdU whereas incubation in $0.1 \mathrm{M}$ sodium citrate buffer, pH6 for $10 \mathrm{~min}$ was done for anti-IL-10, anti-CD3 and anti-CD31. After this, endogenous peroxidase was blocked by $10 \% \mathrm{H}_{2} \mathrm{O}_{2}$-methanol solution for $15 \mathrm{~min}$. Blocking of non-specific background staining were accomplished using 8\% skimmed milk for CD3 and CD31, and Histofine Mousestain Kit (Nichirei Biosciences, Japan) for BrdU. Tissue sections were then subsequently incubated overnight in a humidified chamber at $4{ }^{\circ} \mathrm{C}$ using the following primary antibodies: anti-CD3 (FLEX Polyclonal Rabbit Anti-Human CD3 Ready-to-Use; DakoCytomation, Denmark), anti-CD31 (CD31 Rabbit Polyclonal Antibody Ready-to-Use for Immunohistochemical Staining; Neomarkers, USA) and anti-BrdU (Monoclonal Mouse Anti-Bromodeoxyuridine Clone Bu20a 1:50; DakoCytomation, Denmark). After washing by TBS for 3 times, immunolabeling of tissue sections was performed using Dako EnVision + System-HRP Labelled Polymer Anti-Rabbit (DakoCytomation) for $1 \mathrm{~h}$ under room temperature (CD3 and CD31) or universal immuno-peroxidase polymer (Simple Stain Mouse Max PO; Nichirei Biosciences) for 10 min under room temperature after further blocking by Histofine Mousestain $\mathrm{Kit}$ (BrdU). Immunoreaction was finally visualized using diaminobenzidine tetrahydrochloride $-\mathrm{H}_{2} \mathrm{O}_{2}$ solution. 


\begin{tabular}{|l|l|l|}
\hline Genes & Forward & Reverse \\
\hline Actb & AAGTGTGACGTTGACATCCG & GATCCACATCTGCTGGAAGG \\
\hline$I l l b$ & GCAACTGTTCCTGAACTCAACT & ATCTTTTGGGGTCCGTCAACT \\
\hline$I l 10$ & GCTCTTACTGACTGGCATGAG & CGCAGCTCTAGGAGCATGTG \\
\hline$I l 11$ & TGTTCTCCTAACCCGATCCCT & CAGGAAGCTGCAAAGATCCCA \\
\hline$C x c l 1$ & CTGGGATTCACCTCAAGAACATC & CAGGGTCAAGGCAAGCCTC \\
\hline$C c l 2$ & TTAAAAACCTGGATCGGAACCAA & GCATTAGCTTCAGATTTACGGGT \\
\hline$H g f$ & ATGTGGGGGACCAAACTTCTG & GGATGGCGACATGAAGCAG \\
\hline Egf & AGCATCTCTCGGATTGACCCA & CCTGTCCCGTTAAGGAAAACTCT \\
\hline Fgf7 & CTCTACAGGTCATGCTTCCACC & ACAGAACAGTCTTCTCACCCT \\
\hline$P t g s 2$ & TTCAACACACTCTATCACTGGC & AGAAGCGTTTGCGGTACTCAT \\
\hline
\end{tabular}

Table 2. List of real-time PCR primers.

The number of CD3-, CD31- and BrdU-positive cells was assessed using a defined $100 \mu \mathrm{m}$ area in the pyloric region with the highest cell density and the mean of 8 measurements was obtained.

Quantitative RT-PCR. Another half of harvested gastric pyloric tissues were placed in RNAlater (Invitrogen, USA) and stored under $-80^{\circ} \mathrm{C}$ until further analysis, and quantitative RT-PCR was performed basically the same as previously described ${ }^{24}$. The samples were homogenized with Shake Master Auto BMS-A20TPver.2.0 (BMS, Japan) and RNA was extracted using Nucleospin RNA isolation kit (Macherey-Nagel, Germany) according to the manufacturer's instructions. First strand cDNA was synthesized by PrimeScript RT reagent kit (Perfect Real Time; Takara Bio, Japan). Real-Time PCR analysis was accomplished using TB Green Premix Ex Taq II kit (TIi RNaseH Plus; TaKaRa Bio, Japan) and StepOnePlus Real-Time PCR (Applied Biosystems, USA) to determine the expression level of the following genes which are reported to be affected by A4gnt deficiency in the previous study ${ }^{19}:$ Il1b, Il10, Il11, Cxcl1, Ccl2, Hgf, Egf, Fgf7, Ptgs2, and Actb. The primers utilized in the present study are shown in Table 2. Reaction was heated at $98^{\circ} \mathrm{C}$ for $1 \mathrm{~min}$ followed by 40 thermal cycles at $95^{\circ} \mathrm{C}$ for $30 \mathrm{~s}, 95^{\circ} \mathrm{C}$ for $5 \mathrm{~s}, 60^{\circ} \mathrm{C}$ for $34 \mathrm{~s}$, and one cycle of melting curve at $95^{\circ} \mathrm{C}$ for $15 \mathrm{~s}, 60^{\circ} \mathrm{C}$ for $1 \mathrm{~m}$, and $95^{\circ} \mathrm{C}$ for $15 \mathrm{~s}$. Expression of mRNA was normalized to the expression level of the housekeeping gene, Actb and determination of comparative CT value was done by setting the average mRNA expression level of C57BL/6J mice as 1.0. Analysis was run in duplicates.

IgA concentration of small intestinal contents and tissue. Whole small intestine was harvested and the intestinal contents were washed out using a total of $6 \mathrm{ml}$ of cOmplete (Roche, Switzerland) solution dissolved in $50 \mathrm{ml}$ of PBS and then collected. The intestinal contents were centrifuged at $600 \times G$ for 5 min under $4{ }^{\circ} \mathrm{C}$ and the supernatant was centrifuged again at $9200 \times G$ for $5 \mathrm{~min}$. Two centimeters of ileum about a quarter from the cecum was also collected as small intestinal tissue. The tissue was cut open, washed with PBS and homogenized with $2 \mathrm{ml}$ of homogenization buffer (0.475 g HEPES, $500 \mu \mathrm{l}$ 1:100 Triton X-100 (Sigma, USA), 5 ml glycerol and 1 tablet of cOmplete in $50 \mathrm{ml}$ ). The homogenate was centrifuged at $4000 \mathrm{rpm}$ for $10 \mathrm{~min}$ under $4{ }^{\circ} \mathrm{C}$ and the supernatant was centrifuged again at $7000 \mathrm{rpm}$ for $5 \mathrm{~min}$. IgA concentration of the supernatants were measured with IgA Mouse Uncoated ELISA Kit with Plates (Invitrogen, USA) according to the manufacturer's instructions at $450 \mathrm{~nm}$ using iMark microplate reader (Bio-Rad Laboratories, USA).

Statistical analysis. All values were expressed as mean \pm SD and compared with Tukey's honest significance test using R software ver. 3.6.2 $2^{28}$. Values with $P<0.05$ were considered statistically significant.

Received: 29 June 2020; Accepted: 24 May 2021

Published online: 01 July 2021

\section{References}

1. ILSI. Safety assessment and potential health benefits of food components based on selected scientific criteria. Crit. Rev. Food. Sci. Nutr. 39, 203-206 (1999).

2. Hertog, M. G., Feskens, E. J., Hollman, P. C., Katan, M. B. \& Kromhout, D. Dietary antioxidant flavonoids and risk of coronary heart disease: The Zutphen Elderly Study. Lancet 342, 1007-1011 (1993).

3. Moayyedi, P. et al. The efficacy of probiotics in the treatment of irritable bowel syndrome: A systematic review. Gut 59, 285-286 (2010).

4. Hasler, C. M. Functional foods: Benefits, concerns and challenges-A position paper from the American council of science and health. J. Nutr. 32, 3772-3781 (2002).

5. Shimada, R. et al. Oral administration of green algae, Euglena gracilis, inhibits hyperglycemia in OLETF rats, a model of spontaneous type 2 diabetes. Food Funct. 7, 4655-4659 (2016).

6. Nakashima, A. et al. Oral administration of Euglena gracilis $\mathrm{Z}$ and its carbohydrate storage substance provides survival protection against influenza virus infection in mice. Biochem. Biophys. Biochem. Biophys. Res. Commun. 494, 379-383 (2017). 
7. Nakashima, A. et al. Anti-fibrotic activity of Euglena gracilis and paramylon in a mouse model of non-alcoholic steatohepatitis. Food Sci. Nutr. 7, 139-147 (2019).

8. Nakashima, A. et al. $\beta$-Glucan in foods and its physiological functions. J. Nutr. Sci. Vitaminol. 64, 8-17 (2018).

9. Kondo, Y. et al. Cytokine-related immunopotentiating activities of paramylon, a $\beta-(1 \rightarrow 3)$-D-glucan from Euglena gracilis. J. Pharmacobiodyn. 15, 617-621 (1992).

10. Sugiyama, A. et al. Oral administration of paramylon, a $\beta-1,3-\mathrm{D}$-glucan isolated from Euglena gracilis Z inhibits development of atopic dermatitis-like skin lesions in NC/Nga mice. J. Vet. Med. Sci. 72, 755-763 (2010).

11. Sugiyama, A. et al. Hepatoprotective effects of paramylon, a $\beta$-1,3-D-glucan isolated from Euglena gracilis Z, on acute liver injury induced by carbon tetrachloride in rats. J. Vet. Med. Sci. 71, 885-890 (2009).

12. Koizumi, N. et al. Anti-HIV (human immunodeficiency virus) activity of sulfated paramylon. Antiviral Res. 21, 1-14 (1993).

13. Sakagami, H. et al. Macrophage stimulation activity of antimicrobial N, N-dimethylaminoethylparamylon. In Vivo 5, 101-105 (1991).

14. Brown, G. D. \& Gordon, S. Immune recognition. A new receptor for beta-glucans. Nature 413, 36-37 (2001).

15. Chan, G. C., Chan, W. K. \& Sze, D. M. The effects of beta-glucan on human immune and cancer cells. J. Hematol. Oncol. https:// doi.org/10.1186/1756-8722-2-25 (2009).

16. Watanabe, T. et al. Antitumor activity of the $\beta$-glucan paramylon from Euglena against preneoplastic colonic aberrant crypt foci in mice. Food Funct. 4, 1685-1690 (2013).

17. Panja, S., Ghate, N. B. \& Mandal, N. A microalga, Euglena tuba induces apoptosis and suppresses metastasis in human lung and breast carcinoma cells through ROS-mediated regulation of MAPKs. Cancer Cell Int. 16, 51. https://doi.org/10.1186/s12935-0160330-5 (2016).

18. Nakayama, J. et al. Expression cloning of a human $\alpha-1,4-N$-acetylglucosaminyltransferase that forms GlcNAcal $\rightarrow 4 \mathrm{Gal} \beta \rightarrow \mathrm{R}$, a glycan specifically expressed in gastric gland mucous cell-type mucin. Proc. Nat. Acad. Sci. USA 96, 8991-8996 (1999).

19. Karasawa, F. et al. Essential role of gastric gland mucin in preventing gastric cancer in mice. J. Clin. Investig. 122, 923-934 (2012).

20. Correa, P. \& Piazuelo, M. B. The gastric precancerous cascade. J. Dig. Dis. 13, 2-9 (2012).

21. Lu, H., Ouyang, W. \& Huang, C. Inflammation, a key event in cancer development. Mol. Cancer Res. 4, 221-233 (2006).

22. Grivennikov, S. I., Greten, F. R. \& Karin, M. Immunity, inflammation, and cancer. Cell 140, 883-899 (2010).

23. Ernst, M. et al. STAT3 and STAT1 mediate IL-11-dependent and inflammation-associated gastric tumorigenesis in gp130 receptor mutant mice. J. Clin. Investig. 118, 1727-1738 (2008).

24. Desamero, M. J. et al. Orally administered brown seaweed-derived $\beta$-glucan effectively restrained development of gastric dysplasia in A4gnt KO mice that spontaneously develop gastric adenocarcinoma. Int. Immunopharmacol. 60, 211-220 (2018).

25. Takeyama, H. et al. Production of antioxidant vitamins, $\beta$-carotene, vitamin C and vitamin E, by the two-step cultured Euglena gracilis Z. Biotechnol. Bioeng. 53, 185-190 (1997).

26. Yang, L. et al. A systematic review of the mechanisms underlying treatment of gastric precancerous lesions by traditional Chinese medicine. Evid. Based Complement. Altern. Med. https://doi.org/10.1155/2020/9154738 (2020).

27. Suzuki, K. et al. Selection and characterization of Euglena anabaena var. minor as a new candidate Euglena species for industrial application. Biosci. Biothechnol. Biochem. 79, 1730-1736 (2015).

28. R Core Team. R: A Language and Environment for Statistical Computing https://www.R-project.org/ (R Foundation for Statistical Computing, 2018).

\section{Acknowledgements}

This study was supported by KAKENHI, Grant-in-Aid for Scientific Research on Innovative Areas (Research in a proposed research area) [grant \#23114504] (S.Ka.), and Grant-in-Aid for Challenging Exploratory Research [Grant \#25670191] (S.Ka. and J.N.).

\section{Author contributions}

Conceptualization: S.Ka., K.H. Funding Acquisition: K.S., K.U., J.N., S.Ka., K.H. Investigation: M.I., M.J.D., J.K.C., R.O. Project Administration: S.Ka., K.H. Resources: K.Y., A.N., K.S., J.N. Supervision: K.U., S.H., S.Ky., K.M., S.Ka., K.H. Visualization: M.I., M.J.D., J.K.C. Writing - original draft: M.I., M.J.D., K.H. Writing - review \& editing: K.Y., A.N., K.S., S.H., J.N., S.Ky., K.M., S.Ka., K.H.

\section{Competing interests}

Employment K.Y., A.N. and K.S. are employees of euglena Co., Ltd, the producer of spray-dried powder of Euglena gracilis and paramylon. The other authors (M.I., M.J.D., J.K.C., K.U., R.O., S.H., J.N., S.Ky., K.M., S.Ka. and K.H.) declare no competing interests.

\section{Additional information}

Supplementary Information The online version contains supplementary material available at https://doi.org/ 10.1038/s41598-021-92013-5.

Correspondence and requests for materials should be addressed to S.K. or K.H.

Reprints and permissions information is available at www.nature.com/reprints.

Publisher's note Springer Nature remains neutral with regard to jurisdictional claims in published maps and institutional affiliations.

(c) (i) Open Access This article is licensed under a Creative Commons Attribution 4.0 International License, which permits use, sharing, adaptation, distribution and reproduction in any medium or format, as long as you give appropriate credit to the original author(s) and the source, provide a link to the Creative Commons licence, and indicate if changes were made. The images or other third party material in this article are included in the article's Creative Commons licence, unless indicated otherwise in a credit line to the material. If material is not included in the article's Creative Commons licence and your intended use is not permitted by statutory regulation or exceeds the permitted use, you will need to obtain permission directly from the copyright holder. To view a copy of this licence, visit http://creativecommons.org/licenses/by/4.0/.

(C) The Author(s) 2021 